\title{
Design and Development of Graphene FET Biosensor for the Detection of SARS-CoV-2
}

\section{B. Vamsi Krsihna}

Koneru Lakshmaiah Education Foundation

\section{Shaik Ahmadsaidulu}

Velagapudi Ramakrishna Siddhartha Engineering College

\section{Surapaneni Sai Tarun Teja}

Velagapudi Ramakrishna Siddhartha Engineering College

\section{Jayanthi}

Gokaraju Rangaraju Institute of Engineering and Technology

Alluri Navaneetha

Mahatma Gandhi Institute of Technology

P Rahul Reddy

Geethanjali Institute of Science and Technology

Durga Prakash Matta ( $\sim$ mdprakash82@gmail.com )

Velagapudi Ramakrishna Siddhartha Engineering College https://orcid.org/0000-0001-9143-0628

\section{Research Article}

Keywords: FET, SARS-CoV-2, rGO, Biosensor, Limit of detection.

Posted Date: August 25th, 2021

DOI: https://doi.org/10.21203/rs.3.rs-826470/v1

License: (1) (1) This work is licensed under a Creative Commons Attribution 4.0 International License.

Read Full License 


\title{
Design and Development of Graphene FET Biosensor for the Detection of SARS-CoV-2
}

B. Vamsi Krsihna ${ }^{1}$, Shaik Ahmadsaidulu ${ }^{2}$, Surapaneni Sai Tarun Teja ${ }^{2}$, D Jayanthi ${ }^{3}$, Alluri Navaneetha ${ }^{4}$, P Rahul Reddy ${ }^{5}$ and M. Durga Prakash ${ }^{2, \#}$

${ }^{1}$ Department of ECE, Koneru Lakshmaih Education Foundation, Guntur -522502, Andhra Pradesh, India ${ }^{2}$ Department of ECE, Velagapudi Ramakrishna Siddhartha Engineering College, Kanuru -520007, Andhra Pradesh, India ${ }^{3}$ Department of ECE, Gokaraju Rangaraju Institute of Engineering \& Technology, Hyderabad-500090, Telangana, India ${ }^{4}$ Department of ECE, Mahatma Gandhi Institute of Technology, Hyderabad - 500075, Telangana, India ${ }^{5}$ Department of ECE, Geethanjali Institute of Science and Technology, Nellore-524137, Andhra Pradesh, India

\#Corresponding author E-mail: mdprakasah82@gmail.com

\begin{abstract}
The most affected disease in recent years is Severe Acute Respiratory Syndrome Coronavirus 2 (SARS-COV-2) that is notable as COVID-19. It has been started as a disease in one place and arisen as a pandemic throughout the world. A serious health problem is developed in the lungs due to the effect of this coronavirus. Sometimes it may result in death as a consequence of extensive alveolar damage and progressive respiratory failure. Hence, early detection and appropriate diagnosis of corona virus in patient's body is very essential to save the lives of affected patients This work evolves a Silicon ( $\mathrm{Si}$ ) based label-free electrical device i.e. the reduced graphene oxide field-effect transistor (rGO FET) for SARS-CoV-2 detection. Firstly rGO FET functionalized with SARS-CoV-2 monoclonal antibodies (mAbs). Then the rGO FET characteristic response is observed to detect the antibody-antigen reaction of SARS-CoV-2 with different molar ranges. The developed GFET shows better performance towards the drain current and limit-of-detection (LoD) up to 2E-18 M. Therefore, we believe that an intense response was observed than the earlier developed devices and signifies impressive capability for subsequent implementation in point-of-care (PoC) diagnostic tests.
\end{abstract}

Keywords- FET, SARS-CoV-2, rGO, Biosensor, Limit of detection.

\section{1: Introduction}

Emerging contagious diseases, such as severe acute respiratory syndrome (SARS), Flu, and Ebola infection illness, pose a major risk to human health. In December 2019, a series of patients were admitted to the hospital with symptoms of pneumonia and severe acute respiratory syndrome (SARS) in a place named Wuhan, China. The causes for this disease were unknown initially. From there on, 2019 novel Covid (2019-nCov) was perceived inside the patient's nasal liquid. Subsequently, it was named again as Severe Acute Respiratory Syndrome Coronavirus 2 (SARS-CoV-2) [1,2]. World Health Organization (WHO) alluded to this infection as Corona Virus Disease (Covid-19). It declared that the virus causes this disease as a 2019 novel coronavirus(2019-nCoV). On March eleventh, 2020 WHO recognized this Covid-19 as pandemic as it is transmitting among the people of different countries with rapid speed [3]. The basic symptoms of this disease may include fever, loss of taste or smell, dry cough, and fatigue. The severe symptoms of this disease may contain chest pain or pressure, shortness of breath or difficulty in breathing, and loss of speech or movement. This novel coronavirus is transmitted by droplets and aerosols of human saliva. The severity of this pandemic can be observed by the statistics provided by WHO.

The total number of cases globally is approximately 18.5 crores and the total deaths are 40 Lakhs up to July 1 st week. Due to this pandemic entire world faced different socio-economic problems. To avoid these difficulties, the patients need to be properly diagnosed and treated with utmost care. Even though there are some measures to prevent or control this coronavirus, like WHO-recommended medicines or vaccines but the human life is still having a threat from the Covid-19 pandemic [4-6].

The early detection of this Coronavirus disease in the fluids extracted from patients is utmost prioritized for proper treatment of the patients as this disease may become severe within 5-10 days. In this scenario, laboratory diagnostics is the major parameter to detect the disease even at lower concentrations. To fulfil the above needs, RT-PCR (polymerase chain reaction) is one of the nuclear-derived methods to detect the existence of a particular virus i.e., 2019-nCoV. This technique directly analyses the presence or absence of the virus's RNA. 
Enzyme Linked Immunosorbent Assay (ELISA) analysis is another strategy to distinguish a particular antibody and antigen response. These diagnosis techniques take a longer period of 3 hours to 24 hours for the results. Hence, there is a great need for bio-sensors that can detect the corona virus-like particles and a sensitive and quick method is required to identify the virus particles is required to treat this type of pandemic disease [7-9]. In this study, we design GFET biosensor functionalized mAbs for rapid identification of COVID-19, as shown in Figure 1 .

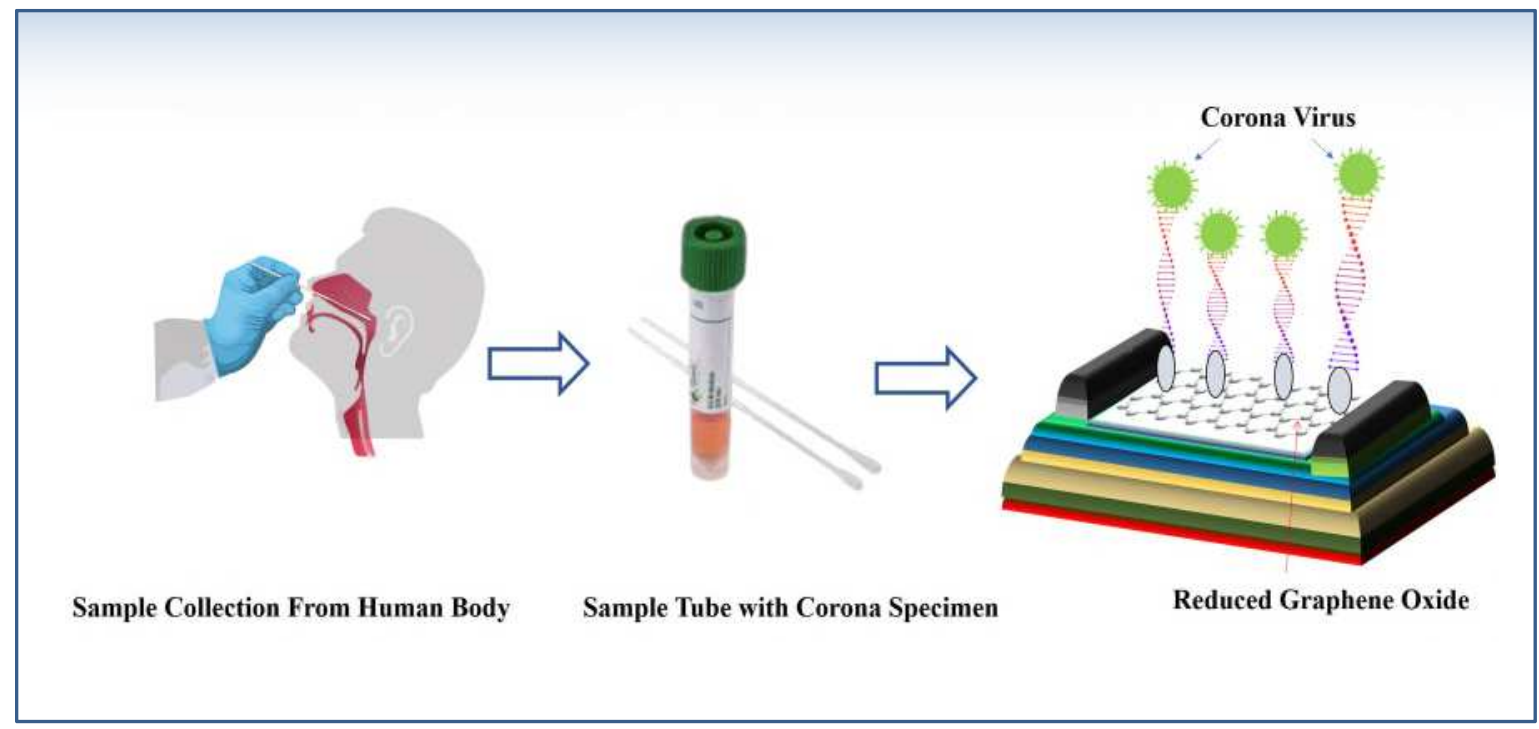

Figure 1: Schematic diagram of SARS-CoV-2 detection using the rGO FET biosensor

The identification involves different steps like collection of samples from the patient, preserving it and finally compelling the sample to get responded with designed GFET. This work mainly aims to identify the presence of novel coronavirus particles through using a GFET and to report its characteristic changes in the presence and absence of coronavirus particles.

\section{2: Design and Simulation of GFET}

A GFET is designed and simulated with the semiconductor module of COMSOL Multiphysics. The dimensions of the designed sensor are gold contact length of $50 \mathrm{~nm}$, the thickness of graphene is $0.35 \mathrm{~nm}$ is used for this design. The silicon dioxide layer with a thickness of $6 \mathrm{~nm}$ acting as a dielectric layer with a dielectric constant of 4.2. The device parameters are shown in Table 1.

The semiconductor module in the software is used for analyzing the transfer characteristics and drain characteristics. The drain current is one of the vital parameters to analyze the enactment of the designed sensor. The drain current flows through the graphene layer only because, it acts as a channel for the conduction of drain current between drain and source and no other channel exists between drain and source. With this, the characteristics of the designed GFET were analyzed without any difficulty.

The electrical characteristics of the designed structure were calculated in the absence and presence of the coronavirus particles. These electrical characteristics are crucial parameters to determine the overall sensing performance of the device. Many important factors affect the electrical characteristics because the drain current varies with different 
parameters of the graphene channel [10]. The GFET designed with the software is shown in Figure 2.

Table 1: Device parameters of GFET

\begin{tabular}{lc}
\hline Device Parameter & Value \\
\hline \hline Thickness of gold contact $\left(\mathrm{t} \_\mathrm{Au}\right)$ & $50 \mathrm{~nm}$ \\
Thickness of $\mathrm{SiO}_{2}\left(\mathrm{t} \_\mathrm{SiO}_{2}\right)$ & $6 \mathrm{~nm}$ \\
Thickness of Silicon (t_Si) & $300 \mathrm{~nm}$ \\
Gold contact length (L_contact) & $500 \mathrm{~nm}$ \\
Drain voltage $\left(\mathrm{V}_{\mathrm{d}}\right)$ & $10 \mathrm{mV}$ \\
Gate voltage $\left(\mathrm{V}_{\mathrm{tg}}\right)$ & $0 \mathrm{~V}$ \\
Source voltage $\left(\mathrm{V}_{\mathrm{s}}\right)$ & $0 \mathrm{~V}$ \\
Width $(\mathrm{W})$ & $4 \mathrm{um}$ \\
Relative permittivity (epsilonr) & 4.2 \\
Donor Concentration & $1 \mathrm{E} 18 \mathrm{~cm}^{-3}$ \\
\hline
\end{tabular}

The software proficiently permits the assignment of different physical properties of the materials to the designed model as per the requirement. The fixed dimensions of GFET allow us to determine the presence and absence of coronavirus antigen protein (spike protein of SARS-CoV-2) along with immobilized monoclonal antibody. With the special characteristics of GFET sensors such as high sensitivity and biological molecule detection, they are making significant progress in the production of ultrasensitive bio-sensors for biochemical analysis. The identification of immobilized antibodies (mAbs) and antigens arise at the graphene channel of the GFET biosensor. The receptor molecule (antibody) is immobilized on the graphene surface which can have the capability to capture the target molecule(antigen).

(a)

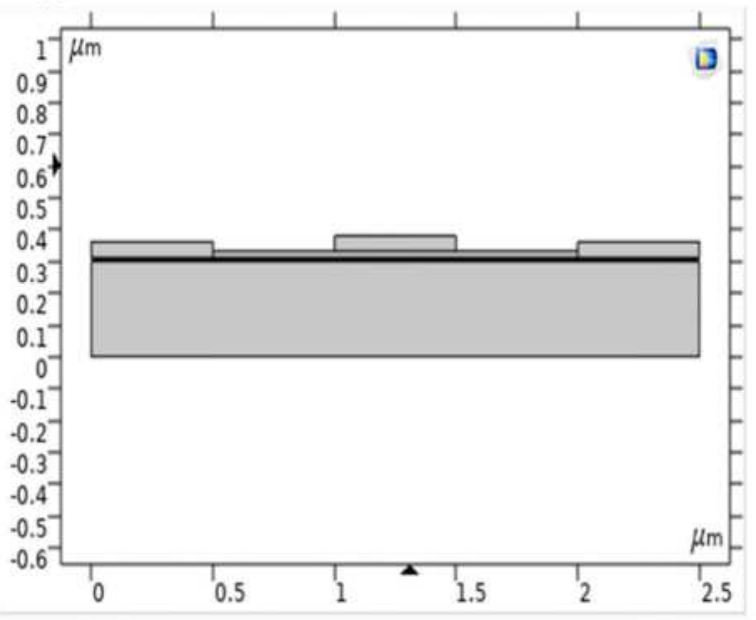

(b)

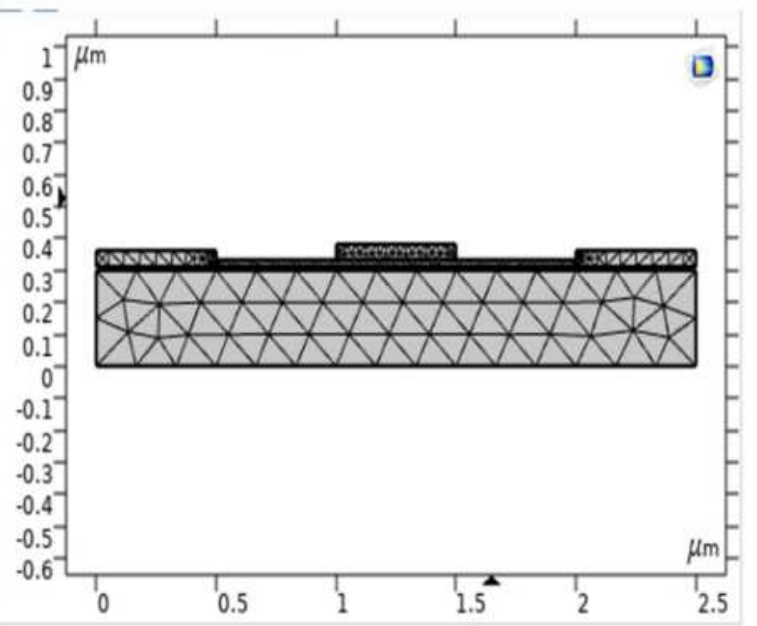

Figure 2: Design structure of (a) GFET (b) Mesh structure of GFET 
The working principle of the GFET biosensor can be described as follows. When a negatively charged antigen, molecule is attached to the surface, the charge carrier's depletion occurs in the complete cross-section of GFET that results in a decrease of electrical conductance and the drain current. Similarly, when a positively charged protein binds to a GFET biosensor, the conductance increases [11-14]. These properties can be demonstrated in COMSOL Multiphysics using the chemical reaction and transport of diluted spices modules, as well as the semiconductor module [15-16]. It can be expressed quantitatively as the concentration of Antigen participating in the reaction with Antibody, as shown in Equation (1).

$$
[k]_{A g}+[k]_{A b} \Leftrightarrow[k]
$$

These antigen particles are ejected from the solution and transported to the immobilized antibodies (mAbs). This can be stated as

$$
[k]_{A B} \Leftrightarrow[k]_{A g}
$$

Where $[k]_{A g}$ denotes the concentration of Antigen-SARS-CoV-2,

$[k]_{A b}$ represents the concentration of SARS-Cov-2 mAb,

$[k] \quad$ signifies the complex concentration.,

$[k]_{A B}$ symbolizes the concentration of SARS-CoV-2 in the bulk fluid.

The modelling was done for low fluid quantities and small-scale designs. As a result, the flow is considered laminar because there is no turbulence and it is a steady flow [17].

$$
\begin{gathered}
\frac{\partial \rho}{\partial t}+\rho(\nabla . U)=0 \\
\rho\left[\frac{\partial U}{\partial t}+U(\nabla . U)\right]=-\nabla P+F+\mu(\nabla . U)
\end{gathered}
$$

Where $\mathrm{P}$ denotes pressure in Pascal, $\mathrm{F}$ denotes body force, $\mathrm{U}$ denotes velocity, $\mu$-dynamic viscosity, and - $\rho$ density. By considering Fick's second law, diffusion of Antigens is expressed as

$$
\frac{\partial C}{\partial t}=\chi \frac{\partial^{2} C}{\partial x^{2}}
$$

In which the term $\partial \mathrm{C} / \partial \mathrm{t}$ is the accumulation which is expressed in $\mathrm{cm}^{-3} \mathrm{~s}^{-1}$,

$\chi$ is the diffusivity in $\mathrm{cm}^{2} / \mathrm{s}$.

The preceding equations are solved using small volume elements to discretize the sensor area. As illustrated in Figure 2(b), the user-defined mesh is used which consisting of tetrahedral or mapped elements with a GFET mesh refinement structure. It was stated that there would be 100 iterations. The mesh variations produced for various element numbers are reassuringly similar. Finalized geometry consists of three domains, sixteen boundaries, sixteen vertices, and twenty-eight edges. The entire mesh comprises 6856 domain elements, 1465 boundary elements, and 386 edge elements.

\section{3: Materials \& Methods}

Graphene was moved to a $\mathrm{SiO} 2 / \mathrm{Si}$ substrate utilizing ordinary wet-move strategies. Poly(methyl methacrylate) (PMMA) C4 (Vision Polymer Pvt., Ltd, Gujarat, India) was turn covered at $500 \mathrm{rpm}$ for $10 \mathrm{~s}$ and at $3000 \mathrm{rpm}$ for 30s onto graphene on $\mathrm{Cu}$ foil (Sigma-Aldrich, Bangalore, India). Poly (methyl methacrylate) (PMMA) C4 (Vision Polymer Pvt., Ltd, Gujarat, India) was turn covered at $500 \mathrm{rpm}$ for $10 \mathrm{~s}$ and $3000 \mathrm{rpm}$ for $30 \mathrm{~s}$ onto graphene on $\mathrm{Cu}$ 
foil (Sigma-Aldrich, Bangalore, India). PMMA/graphene on $\mathrm{Cu}$ foil was scratched in CE-100 copper etchant (MG chemicals, India). After the $\mathrm{Cu}$ foil was scratched, the PMMA/graphene layers were moved to utilize clean glass slides into a deionized (DI) water (Astraa chemicals, Chennai, India), and the copper etchant was laved. Consequently, the $\mathrm{PMMA} /$ graphene layer was moved to a $\mathrm{SiO} 2 / \mathrm{Si}$ substrate and dried under surrounding conditions for the time being. To make useful graphene-based devices, the graphene was patterned into required shapes by the photolithography method Figure 3 (a-c).

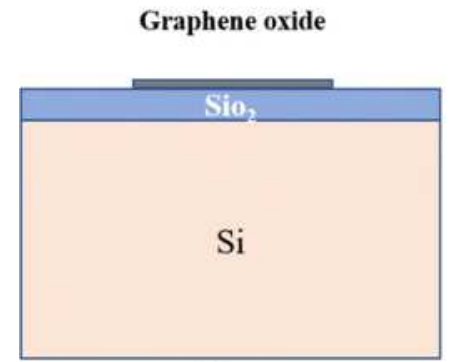

(a)

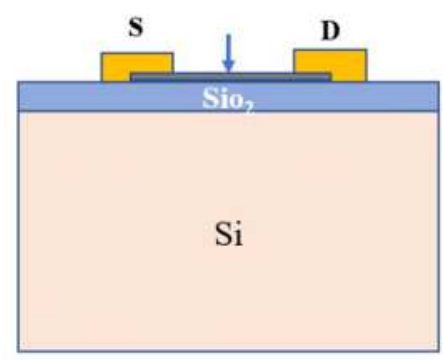

(d)

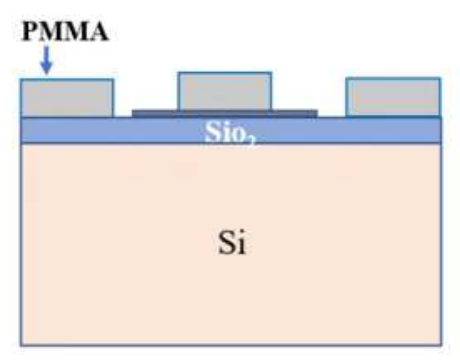

(b)

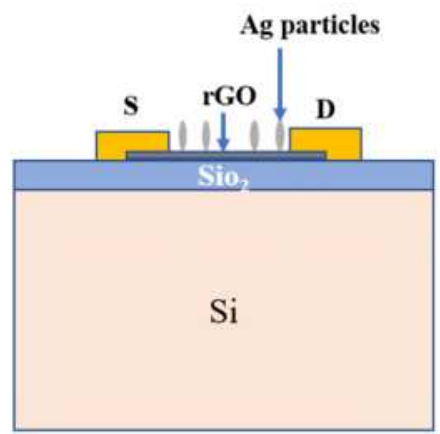

(e)

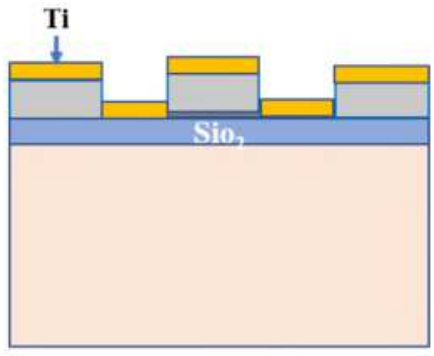

(c)

Figure 3: Fabrication flow GFET, (a) Graphene oxide placed on Silicon Substrate, (b) PMMA coating acts as resist, (c) Physical Vapour Deposition of Titanium, (d) Lift-off of Titanium identify terminals of GFET, (e) rGO by chemical reduction technique with hydrazine

Arbitrary nature of shed graphene made utilizing of EBL inescapable in GFET manufacture measure. One-step and two-steps e-shaft lithography is applied for back-gate and top-gate gadgets separately. Roughly 200 nm PMMA (A2) was spinned as EBL oppose, and was uncovered by Raith Turnkey 150 SEM and E-shaft lithography framework. The rGO was ready by chemical reduction technique with hydrazine, which is considered as standard reductant for GO. Hydrazine can successfully eliminate the oxygen-containing gatherings [18] Figure 3 (d-e). Immobilization is also one of the important steps to analyze the characteristics of the designed GFET as it is to be equipped with SARSCoV-2-Spike protein C-terminal antibody (Sigma-Aldrich, Bangalore, India) to identify the respective SARS-CoV-2 antigens (Sigma-Aldrich, Bangalore, India).

In GFET bio-sensors, dopped channels are substituted with graphene material and these gate terminals are replaced with bio-receptors. Despite the differences in structure, GFET sensors work in the same way as regular FET sensors. The conductivity of GFET is changed by charged particles that are attached to them. The oxide layer acts as an isolation layer on the Si surface of bio-sensors. Even though charged biomolecules accumulated on the oxide layer, the conductivity of the sensor will not be affected [19-22]. Pure graphene is not that sensitive to charged bioparticles. Hence, the surface of GFET must be functionalized. The functionalization may include receptors are to be attached to 
these sensors for the detecting of the specifically charged biomolecules (target molecules). With this, chemical connections between the surface of graphene and biomolecules can be established. The receptors along with target molecules, create an electric field on the surface of the GFET bio-sensor and which will bring a considerable change in the conductivity of the sensor.

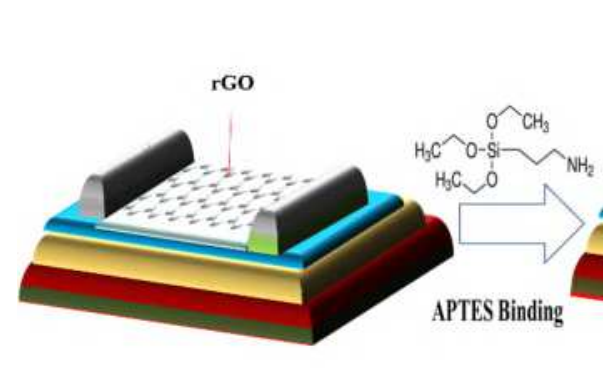

(a)

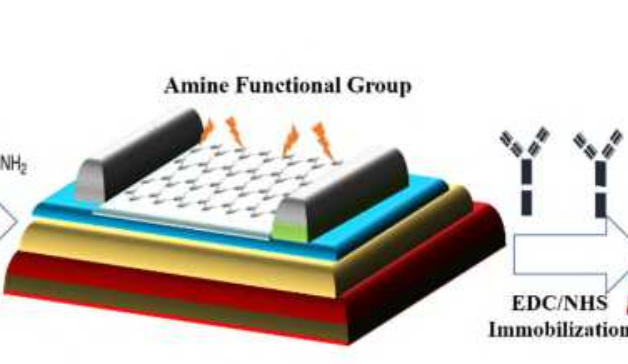

(b)

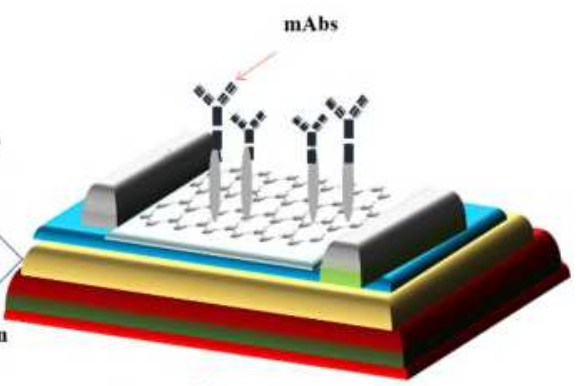

(c)

Figure 4: Procedural steps for immobilization of SARS-CoV-2 mAb: (a) Reduced graphene oxide FET (b) GFET with amine functional group on the graphene surface through APTES binding and (c) Immobilization of mAbs with EDC/NHS

When we consider receptors along with target particles as an input, the receptors function similar to that of the gate (as they are on the surface of graphene) in that they convert the input signal to bring changes in the conductivity of the sensor [23]. Hence, one of the most essential factors in identifying target particles (SARS-CoV-2 Antigen) is the functionalization of GFET. For this immobilization process, firstly GFET sensor was salinized with Glutaraldehyde. Further, it is treated with EDC (1-ethyl-3-(3-dimethylamino) propyl carbodiimide, hydrochloride (Sigma-Aldrich, Bangalore, India), and Sulfo-NHS (Sigma-Aldrich, Bangalore, India) to make mAb immobilized as shown in the Figure 4.

We must equip GFET with receptor particles (SARS-CoV-2 mAb) to identify the target molecules. Figure 4 depicts the general binding process using 3-aminopropyltriethoxysilane (APTES), which acts as a receptor [24-25]. APTES breaks down silicon-oxygen bonds to form a layer in which the specific receptor is present. Immobilization of receptor particles on the surface of the sensor starts with salinization of the sensor with Glutaraldehyde. Afterward, EDC (1ethyl-3-(3-dimethylamino) propyl carbodiimide, Hydrochloride, and Sulfo-NHS are used for protein cross-linking and immobilization. The electrical characteristics of the designed sensor are calculated. Later on, they compared the characteristics of the sensor with the presence of SARS-CoV-2 mAb and in the non-existence of coronavirus particles (SARS-CoV-2). The conductance is relatively more when compared to the bare GFET conductance. The current is increased when $\mathrm{mAb}$ is attached to the GFET sensor. The results obtained were discussed in the next section.

\section{Results and Discussion}

\subsection{Morphological Characterization}

To affirm that the GFET biosensor was perfectly designed, definite characterization was performed after each progression of functionalization. The scanning electron microscopy (SEM) used for validation, which introduced a homogeneous and high thickness of rGO surface. Image of rGO sheets as seen through a scanning electron microscope shown in Figure 5(a). rGO sheets which are having less layers have been stacked and attempted to limit the wrinkles and foldings by utilizing transmission microscope (TEM). The TEM image of rGO sheet was shown in Figure 5(b), which has layers of the order $(n<6)$. HRTEM micrograph of rGO sheets and it plainly shows the grid edges of graphene. This gives extra data about the interplanar distance d002 for rGO material which worth is $3.850 \AA$. The crystallographic design of the graphene sheets was portrayed by selected area electron diffraction (SAED) technique. The past investigations referenced that the majority of the graphene sheets showed a solitary arrangement of hexagonal diffraction design with sharp and clear diffraction spots $[\mathrm{x}]$ as shown Figure 5(c). SAED pattern of 
reduced graphene oxide. The obtained SAED data show unambiguously that the sample RGO differs from Graphite 2H PDF 751621 and has typical interplanar distance d002 from $3.586 \AA$ up to $4.016 \AA$ for three different RGO samples Figure 5(d).
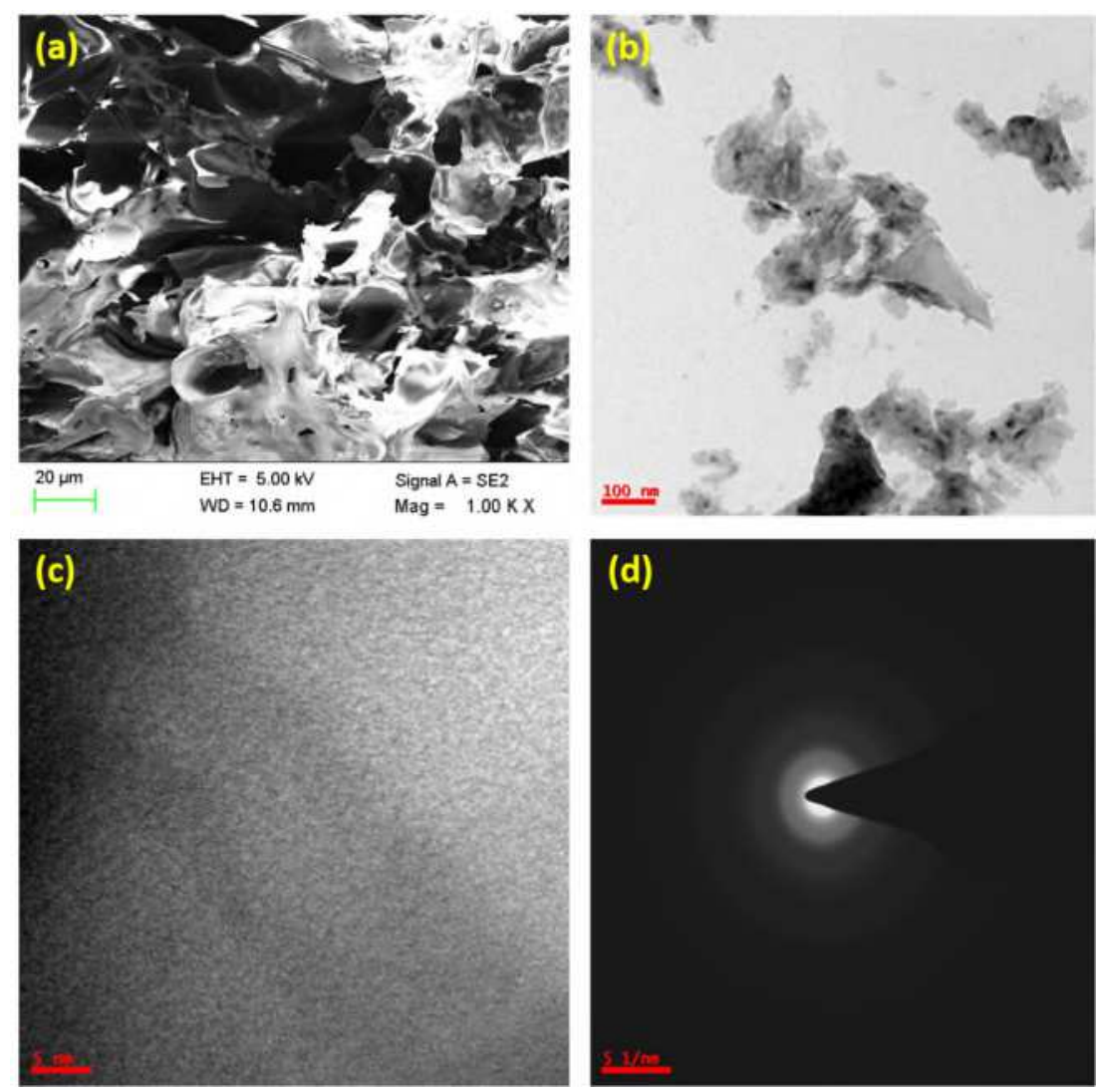

Figure 5: Images of reduced graphene oxide nanocomposites: (a) SEM (b) TEM (c) HRTEM and (d) SAED

\subsection{Electrical Characterization}

To determine the electrical characteristics, the thickness of silicon is considered as 300nm the thickness of silicon dioxide is considered as $6 \mathrm{~nm}$. The conductance of the channel is assessed according to the available charging carriers, which are affected by the voltage of the gate. In return, the driving controls the current of the drain terminal. The drain $\left(\mathrm{I}_{\mathrm{d}}\right)$ is measured with a constant drain voltage of $2 \mathrm{~V}, 4 \mathrm{~V}$, and $6 \mathrm{~V}$ when the source is connected to the ground. The drain current $\left(\mathrm{I}_{\mathrm{d}}\right)$ is calculated in the drain characteristics by sweeping the drain voltage from 0 to $20 \mathrm{~V}$ at various constant gate voltages $\left(\mathrm{V}_{\mathrm{g}}\right)$ such as $6 \mathrm{~V}, 8 \mathrm{~V}$, and $1 \mathrm{~V}$. The results of the simulation are very similar to the results of the experimental sensor in terms of accuracy for both characteristics as shown in Figure 6(a) \& (b)

A current of the nano-ampere order comes with the characteristics of the bare GFET. With small voltage sizes, the IV special behavior of the GFET utilizes the ability to identify charged molecules (present at the surface of the sensor) with detectable current changes. The small change in the drain current along the graphene gives an overview of the $I_{d}-V_{d}$ curve. The biosensor was used to detect coronavirus antigens by the measurement of current in the circuit The GFET is exposed to mAbs and antigens with which analyzed the performance characteristic changes to be applied voltages. To perform this analysis, the drain voltage is varied in the range of 0 to $50 \mathrm{~V}$, with the different concentrations of SARS-CoV-2 like 2*10E-13M, 2*10E-15M, and 2*10E-18M. An adsorption concentration of one molecule per square nanometer of the biomarker was considered on the surface of the gate. 
(a)

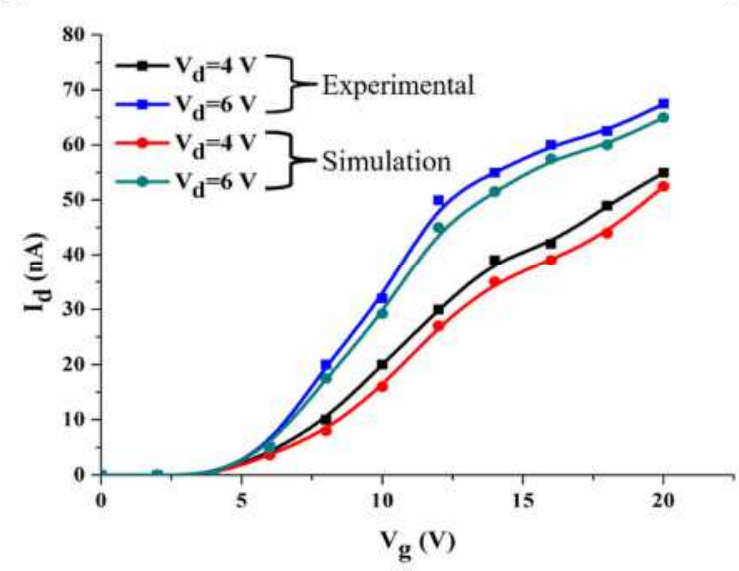

(b)

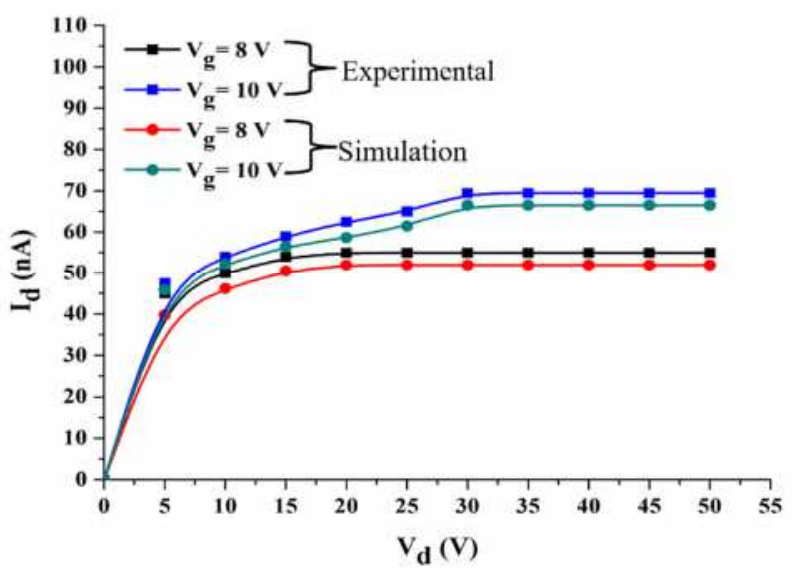

Figure 6: Electrical characteristics of the GFET (a) $V_{g}-I_{d}$ (b) $V_{d}-I_{d}$

In this analysis, a linear dependence of the transistor's current value on the tested quantity of antigens is observed. The Biosensor Current is modulated by antibody-antigens bindings as specific protein is on the surface. The drain characteristics are evaluated in the presence of SARS-CoV-2mAb to check the sensitivity of this GFET about the coronavirus particles, compared to a reference test in the absence of SARS-CoV-2. When SARS-CoV-2 exists concerning the design parameters, the designed GFET changes its drainage current. The maximum current without any virus particle is 70 nano-amperes, whilst the maximum drain current raises to 170 nano-amperes in the presence of antigen.

(a)

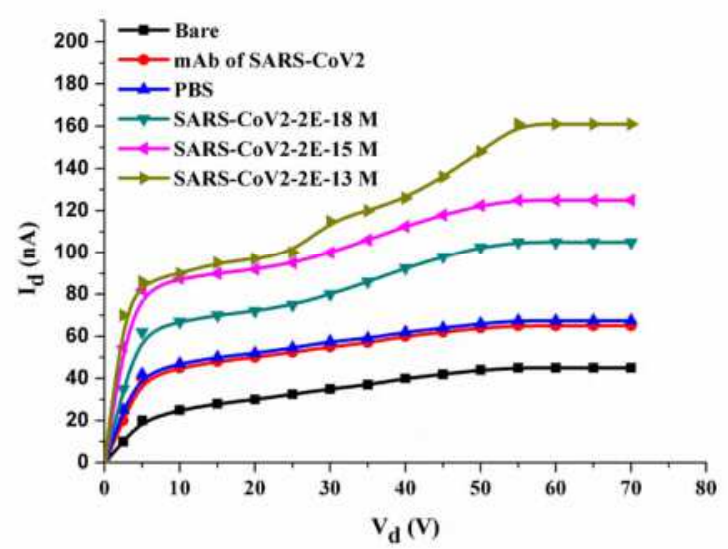

(b)

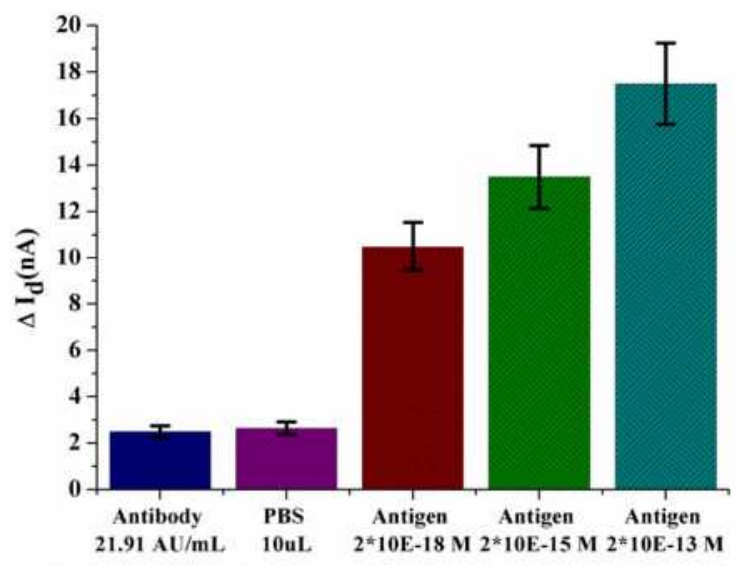

Figure 7: Analytical characteristics of GFET (a) Drain current with different concentrations of antigen (b) change in drain current with different concentrations of antigen

Firstly, the drain current of the designed sensor without mAbs has been calculated. The order of the 70-nano-ampere is then observed in the current as shown in Figure 7(a). The current raised to 50 nano-amperes by load accumulated because of antibody present when it was fitted with SARS-CoV-2 MAb. Furthermore, when the sensor reacted with PBS solution, there is no current change observed. The sensor is subsequently equipped with different antigen concentrations, then the current is amplified according to SARS-Cov-2 antigens. The current is shown as $170 \mathrm{nA}$ for the $2 \mathrm{E} 10-18 \mathrm{M}$ concentration. The change drain current $\left(\Delta \mathrm{I}_{\mathrm{d}}\right)$ with an error $( \pm 10 \%)$ value, with respect to different concentrations of antigen is shown in Figure 7 (b). The maximum current change is observed with antigen of concentration of $2 \mathrm{E}-13 \mathrm{M}$ which the order of $18 \mathrm{nA}$ which is 9 times of antibody concentration's current change with which we can conclude that antigen with higher concentration leads to higher current changes. Based on 3-fold of 
signal to noise ratio, the limit of detection was determined to be $0.002 \mathrm{fM}$. Additionally, Figure 7(b) gives a more instinctive show and comparison. As we can observe, the detecting signals delivered with various concentrations of samples were stronger than those in PBS at a similar concentration of target, in comparison with the background baseline (3-fold of blank), with satisfactory detection limits, they were still significant and measurable. The comparative analysis of different FETs based platforms for the detection of various virus diseases is shown in Table 2. Among all the designed GFET shown excellent limit of detection value.

Table 2: Limit of detection comparison with various FET immunosensors .

\begin{tabular}{cccc}
\hline Virus Type & $\begin{array}{c}\text { Type of Immunosensor } \\
\text { FET }\end{array}$ & Limit of detection & Ref. \\
\hline Hepatitis-B Virus & Tailored DG FET & $1.5 \mathrm{fM}$ & {$[26]$} \\
\hline $\begin{array}{c}\text { SARS-CoV N } \\
\text { protein }\end{array}$ & $\begin{array}{c}\text { Carbon nanotube FET } \\
(\text { CNTFET })\end{array}$ & $5 \mathrm{nM}$ & {$[27]$} \\
\hline SARS-CoV N & High-Electron Mobility & & {$[28]$} \\
protein & Transistor FET (HEMT) & $0.003 \mathrm{nM}$ & {$[29]$} \\
\hline SARS-CoV-2 & SiNW FET & $1 \mathrm{fM}$ & {$[30]$} \\
\hline SARS-CoV-2 & Graphene & $0.2 \mathrm{pM}$ & This Work \\
\hline SARS-CoV-2 & Graphene & $0.002 \mathrm{fM}$ & \\
\hline
\end{tabular}

\section{Conclusion}

In this work, reduced Graphene FET was designed and characterized. The GFET characteristics were observed in different extents. These device characteristics are sensitive to charged biomolecules. This study shows that the presence of the SARS-CoV-2 virus (antibody-antigen binding), greatly affects the electrical properties of the designed GFET so as the sensor characteristics. The device is sensitive to biomolecules adsorbed by the receptor. Very diluted coronavirus detections were obtained at $2 \mathrm{E}-18 \mathrm{M}$. The designed GFET is a strong device to rapidly screen virus proteins, small biomolecules, and different biomarkers. These biosensors are especially advanced for future biomedical applications, such as diagnosis of virus infection and lab-on-chip platforms also.

\section{Acknowledgment}

The authors would like to acknowledge Indian Institute of Technology Hyderabad (IIT Hyderabad) for backing us with some experimental work carried out and the tool (COMSOL Semiconductor Module) required for simulating this work. And M. Durga Prakash thankfully acknowledges this publication as an outcome of the R\&D work undertaken project under the Start-up Research Grant (File No.: SRG/2019/002236) scheme of Department of Science and Technology (DST), Government of India, being Science Engineering Research Broad (SERB).

\section{Funding statement}

The authors did not receive support from any organization for the submitted work. 


\section{Conflict of Interest}

The authors declare that they have no conflict of interest.

\section{Author contributions}

M. Durga Prakash, B. Vamsi Krsihna and Shaik Ahmadsaidulu: Conceptualization; M. Durga Prakash, B. Vamsi Krsihna and Shaik Ahmadsaidulu: investigation; M. Durga Prakash, Shaik Ahmadsaidulu, B. Vamsi Krsihna, and B: resources; M. Durga Prakash, Shaik Ahmadsaidulu, B. Vamsi Krsihna and Surapaneni Sai Tarun Teja, D Jayanthi, Alluri Navaneetha and P Rahul Reddy: data curation; M. Durga Prakash, Surapaneni Sai Tarun Teja, D Jayanthi, Alluri Navaneetha, P Rahul Reddy and Shaik Ahmadsaidulu: writing_original draft preparation; M. Durga Prakash,

B. Vamsi Krsihna, and Shaik Ahmadsaidulu: writing_review and editing; M. Durga Prakash and Shaik Ahmadsaidulu: visualization; M. Durga Prakash: supervision;

\section{Availability of data and material}

No supplementary materials

\section{Compliance with ethical standards}

This article does not contain any studies with human or animal subjects.

\section{Consent to participate}

Additional informed consent was obtained from M. Durga Prakash identifying information is included in this article.

\section{Consent for Publication}

Author(s): Dr. M. Durga Prakash

Author's signature:

\section{Frakash}

Dr. M. Durga Prakash (

Date: 18-08-2021.

\section{REFERENCES}

[1] Wu F, Zhao S, Yu B, Chen YM, Wang W, Song ZG, Hu Y, Tao ZW, Tian JH, Pei YY, Yuan ML. “A new coronavirus associated with human respiratory disease in China." Nature 579, 265-269 (2020). https://doi.org/10.1038/s41586-020-2008-3

[2] WHO. Novel Coronavirus (2019-nCoV) Situation Report-1. https://www.who.int/docs/defaultsource/coronaviruse/situationreports/20200121-sitrep-1-2019-ncov.pdf?sfvrsn=20a99c10_4(2020-04-15).

[3] Coronavirus Disease (COVID-19) Pandemic. Available online: https://www.who.int/ru/emergencies/ diseases/novel-coronavirus-2019 (accessed on 1 July, 2021).

[4] Jayaweera M, Perera H, Gunawardana B, Manatunge J. "Transmission of COVID-19 virus by droplets and aerosols: A critical review on the unresolved dichotomy." Environmental Research 188 (2020): 109819. https://doi.org/10.1016/j.envres.2020.109819.

[5] https://covid19.who.int/table 
[6] Amira Echtioui, Wassim Zouch, Mohamed Ghorbel, Chokri Mhiri, Habib Hamam. "Detection Methods of COVID-19.”, Translating Life Sciences Innovation,25(6), 566-572, https://doi.org/10.1177\%2F2472630320962002

[7] https://www.iaea.org/newscenter/news/how-is-the-covid-19-virus-detected-using-real-time-rt-pcr

[8] FroylaÂn Ibarra, , Natividad Montenegro, Yolanda Vera, Chantal Boulard, Hector Quiroz, Jaime Flores, Pedro Ochoa. "Comparison of three ELISA tests for seroepidemiology of bovine fascioliosis" Veterinary Parasitology ,1998, Vol.77, 229-236. https://doi.org/10.1016/S0304-4017(98)00111-3.

[9] : B. V. Krsihna, S. Ravi and M. D. Prakash, Recent developments in graphene based field effect transistors, Materials Today: Proceedings, https://doi.org/10.1016/j.matpr.2020.07.678

[10] Shaik Ahmadsaidulu, M. Durga Prakash "Impacts of gate length and doping concentrations on the performance of silicon nanowire Field effect Transistor”, Materials Today: Proceedings, Volume 46, Part 9,

2021, Pages 3693-3698. https://doi.org/10.1016/j.matpr.2021.01.849.

[11] Durga Prakash Matta, Siva Rama Krishna Vanjari, Chandra Shekhar Sharma, and Shiv Govind Singh. "Ultrasensitive, label free, chemiresistive nanobiosensor using multiwalled carbon nanotubes embedded electrospun SU-8 nanofibers.” Sensors 16, no. 9 (2016): 1354. https://doi.org/10.3390/s16091354.

[12] Matta Durga Prakash, Suryasnata Tripathy, Siva Rama Krishna Vanjari, Chandra Shekhar Sharma, and Shiv Govind Singh. "An ultrasensitive label free nanobiosensor platform for the detection of cardiac biomarkers." Biomedical microdevices 18, no. 6 (2016): 1-10. https://doi.org/10.1007/s10544-016-0126-3.

[13] Fan Yang, Guo-Jun Zhang. "Silicon nanowire-transistor biosensor for study of molecule-molecule interactions". Reviews in Analytical Chemistry, vol. 33, no. 2, 2014, pp. 95-110.

https://doi.org/10.1515/revac-2014-0010.

[14] Fernando Patolsky, Brian P. Timko, Gengfeng Zheng, and Charles M. Lieber. "Nanowire-based nanoelectronic devices in the life sciences." MRS bulletin 32, no. 2 (2007): 142-149.

https://doi.org/10.1557/mrs2007.47.

[15] Tabatabaian M. CFD module. Stylus Publishing, LLC, 2015.

[16] Multiphysics, Comsol. “Chemical Reaction Engineering Module User’s Guide.” COMSOL AB (2015).

[17] Hajji, L. Kolsi, W. Hassen, A.A.A.A. Al-Rashed, M.N. Borjini, M.A. Aichouni. "Finite element simulation of antigen-antibody transport and adsorption in a microfluidic chip." Physica E: Low-dimensional Systems and Nanostructures 104 (2018): 177-186. https://doi.org/10.1016/j.physe.2018.07.034.

[18] Adriano Ambrosi, Chun Kiang Chua, Alessandra Bonanni, and Martin Pumera, "Lithium Aluminum Hydride as Reducing Agent for Chemically Reduced Graphene Oxides”, Chemistry of Materials, 2012, 24, 12, $2292-2298$. https://doi.org/10.1021/cm300382b

[19] R.L. Puurunen, "Surface chemistry of atomic layer deposition: A case study for the trimethylaluminum/water process,” Journal of Applied Physics, vol. 97, 2005, p. 121301. https://doi.org/10.1063/1.1940727

[20] Mo-Yuan Shen, Bor-Ran Li and Yaw-Kuen Li. "Silicon nanowire field-effect-transistor based biosensors: From sensitive to ultra-sensitive.” Biosensors and Bioelectronics, Volume 60 (2014): 101-111. https://doi.org/10.1016/j.bios.2014.03.057.

[21] Charles M. Lieber. "Nanoscale Science and Technology: Building a Big Future from Small Things.” MRS Bulletin, Volume 28(07), pp. 486-491. https://doi.org/10.1557/mrs2003.144.

[22] Yue Wu, Jie Xiang, Chen Yang, Wei Lu \& Charles M. Lieber. "Single-crystal metallic nanowires and metal/semiconductor nanowire heterostructures." Nature, 430(6995), pp.61-65.

[23] G. Zheng, W. Lu, S. Jin, C. M. Lieber. "Synthesis and fabrication of high-performance n-type silicon nanowire 
transistors.” Advanced Materials 16, no. 21 (2004): 1890-1893.

[24] Yi Cui, Qingqiao Wei, Hongkun Park and Charles M. Lieber, "Nanowire nanosensors for highly sensitive and selective detection of biological and chemical species.” science 293, no. 5533 (2001): 1289-1292.

[25] Chunyu Chan et al., 'A Microfluidic Flow-through Chip Integrated with Reduced Graphene Oxide Transistor for Influenza Virus Gene Detection', Sensors and Actuators B: Chemical 251 (1November 2017): 927-33, https://doi.org/10.1016/j.snb.2017.05.147.

[26] I.-K. Lee, M. Jeun, H.-J. Jang, W.-J. Cho and K. H. Lee, “A self-amplified transistor immunosensor under dual gate operation: highly sensitive detection of hepatitis B surface antigen", Nanoscale, 2015,7, 16789-16797, https://doi.org/10.1039/C5NR03146J.

[27] F.N. Ishikawa, M. Curreli, C.A. Olson, H.I. Liao, R. Sun, R.W. Roberts, R.J. Cote, M.E. Thompson, C. Zhou, "Importance of Controlling Nanotube Density for Highly Sensitive and Reliable Biosensors Functional in Physiological Conditions", ACS Nano 4 (2010) 6914-6922, https://doi.org/10.1021/nn101198u

[28] Y.-R. Hsu, G.-Y. Lee, J.-I. Chyi, C.-k. Chang, C.-C. Huang, C.-P. Hsu, T.-h. Huang, F. Ren, 681 Y.-L. Wang, "Detection of Severe Acute Respiratory Syndrome (SARS) Coronavirus Nucleocapsid 682 Protein Using AlGaN/GaN High Electron Mobility Transistors", ECS Trans. 50 (2013) 239-243, https://doi.org/10.1149/05006.0239ecst

[29] Jay Huiyi Chua, Ru-Ern Chee, Ajay Agarwal, She Mein Wong, and Guo-Jun Zhang. "Label-free electrical detection of cardiac biomarker with complementary metal-oxide semiconductor-compatible silicon nanowire sensor arrays.” Analytical chemistry 81, no. 15 (2009): 6266-6271. https://doi.org/10.1021/ac901157x.

[30] Xiaoyan Zhang et al., 'Electrical Probing of COVID-19 Spike Protein Receptor Binding Domain via a Graphene Field-Effect Transistor', ArXiv:2003.12529 [Cond-Mat, Physics: Physics], 27 March 2020, http://arxiv.org/abs/2003.12529. 
